\title{
Effects of Breathing Exercises in Patients With Chronic Obstructive Pulmonary Disease: Systematic Review and Meta-Analysis
}

\author{
Nutsupa Ubolnuar, PT, BSc ${ }^{1}$, Anong Tantisuwat, PT, PhD $^{1}$, Premtip Thaveeratitham, PT, $\mathrm{PhD}{ }^{1}$, \\ Somrat Lertmaharit, $\mathrm{MSc}^{2}$, Chathipat Kruapanich, PT, $\mathrm{MSc}^{1}$, Witaya Mathiyakom, PT, $\mathrm{PhD}^{3}$ \\ ${ }^{1}$ Department of Physical Therapy, Faculty of Allied Health Sciences, Chulalongkorn University, Bangkok; \\ ${ }^{2}$ Department of Preventive and Social Medicine, Faculty of Medicine, Chulalongkorn University, Bangkok, Thailand; \\ ${ }^{3}$ Department of Physical Therapy, California State University, Northridge, CA, USA
}

Objective To update evidence on the effects of breathing exercises (BEs) on ventilation, exercise capacity, dyspnea, and quality of life (QoL) in chronic obstructive pulmonary disease (COPD) patients.

Methods Randomized controlled trials investigating the effects of BEs in COPD patients published through May 2018, were retrieved from five electronic databases (MEDLINE, CINAHL, Cochrane, Scopus, and ScienceDirect). Risk of bias and quality of evidence were assessed, using Cochrane Collaboration's tool, and the Grading of Recommendation Assessment, Development, and Evaluation (GRADE) approach, respectively.

Results Nineteen studies ( $\mathrm{n}=745)$, were included. Quality of evidence, was low to moderate. When compared to the control groups, respiratory rate significantly $(\mathrm{p} \leq 0.001)$ improved in the pursed-lip breathing (PLB), ventilatory feedback (VF) plus exercise, diaphragmatic breathing exercise (DBE), and combined BEs. Additionally, PLB significantly improved tidal volume $(\mathrm{p}<0.001)$, inspiratory time $(\mathrm{p}=0.007)$, and total respiratory time $(\mathrm{p}<0.001)$. VF plus exercise significantly improved inspiratory capacity $(\mathrm{p}<0.001)$, and singing significantly improved the physical component of QoL, than did the control groups $(\mathrm{p}<0.001)$. All BEs did not significantly improve dyspnea, compared to the controls ( $\mathrm{p}>0.05$ ).

Conclusion PLB, VF plus exercise, DBE, combined BEs, and singing could be used to improve ventilation and QoL. Based on low to moderate quality of evidence, use of these BEs to improve ventilation and QoL in COPD patients is conditional (Registration No. CRD42018102995).

Keywords Breathing exercises, Ventilation, Dyspnea, Chronic obstructive pulmonary disease, Meta-analysis

Received November 15, 2018; Accepted February 28, 2019

Corresponding author: Anong Tantisuwat

Department of Physical Therapy, Faculty of Allied Health Sciences, Chulalongkorn University, 154 Rama I Road, Pathumwan, Bangkok 10330, Thailand. Tel: +66-2-218-3768, Fax: +66-2-218-3766, E-mail: anong.ta@chula.ac.th

ORCID: Nutsupa Ubolnuar (https://orcid.org/0000-0002-5072-9559); Anong Tantisuwat (https://orcid.org/0000-0003-3845-932X); Premtip Thaveeratitham (https://orcid.org/0000-0002-6768-2363); Somrat Lertmaharit (https://orcid.org/0000-0002-8179-7570); Chathipat Kruapanich (https://orcid.org/0000-0001-5795-8138); Witaya Mathiyakom (https://orcid.org/0000-0002-4801-7317).

(a) This is an open-access article distributed under the terms of the Creative Commons Attribution Non-Commercial License (http://creativecommons.org/ licenses/by-nc/4.0) which permits unrestricted noncommercial use, distribution, and reproduction in any medium, provided the original work is properly cited. Copyright ( 2019 by Korean Academy of Rehabilitation Medicine 


\section{INTRODUCTION}

Chronic obstructive pulmonary disease (COPD) is one of the most common life-threatening diseases affecting populations globally [1]. Pathophysiological changes in airway, tissue, and vascular supply to lungs increase airway resistance and air trapping, and decrease lung compliance resulting in increased work of breath, and dyspnea in COPD patients [2]. To avoid dyspnea, COPD patients commonly avoid or limit physical activities which, in turn, lead to decrease in exercise tolerance, and an increase in anxiety, disability, and poor quality of life (QoL) [3-5]. So, comprehensive management including interventions to relieve sensation of dyspnea to improve exercise tolerance and QoL, is needed in this population [6].

Breathing exercise (BE) has been an essential part of a comprehensive pulmonary rehabilitation program, for COPD patients. Many types of BE such as slow and deep breathing, active expiration, pursed-lip breathing (PLB), relaxation breathing, diaphragmatic breathing (DBE), and ventilatory feedback (VF) training, have been prescribed to decrease lung hyperventilation, enhance respiratory muscle function, exercise tolerance, and QoL in COPD patients [7]. These BEs have been used individually, or in combination of different types of BE [7].

Although systematic reviews (SRs) [8-11] and a review of literature [12] have suggested the effects of several types of $\mathrm{BE}$ in patients with mild to very severe COPD, the effects of BEs remain inconclusive due to inconsistency of results across studies, as well as limited quality of evidence. For example, PLB was shown to improve ventilation, exercise endurance, dyspnea, and QoL $[8,9,11]$. However, the effects of PLB on these outcomes cannot be confirmed, because of low quality of SR [8], limited data to perform meta-analysis [8], inconsistent results across trials $[8,9]$, and small numbers of included studies $[8,9]$. In a recent SR published in 2018, PLB was shown to significantly improve ventilation-related outcomes such as respiratory rate and minute ventilation, but not dyspnea and exercise capacity [11]. However, results were derived from studies with poor to fair quality as indicated by PEDro scores, and the quality of evidence of each outcome was not systematically assessed. These two limitations negatively impact, the decision of using PLB in clinical setting [11]. Singing was shown, in a SR, to significantly improve QoL and anxiety in COPD patients [10]. However, the SR suffered from a small number of included studies, heterogeneity in results among studies, and qualitative analysis used in the study [10]. The effects of DBE are shown to be inconclusive, between studies $[9,12]$. For example, in the previous SR, DBE was shown to improve exercise endurance and QoL [9]. In contrast, results of review literature revealed conflicting effects of DBE, on dyspnea and ventilation [12]. However, the effects of DBE were concluded from only one study [9] and review of literature, without assessing the quality of included studies [12]. Large variation in the effects BEs noted between studies of the same types of BE as well as between different types of BE, impedes clinicians' ability to make a clear decision, when prescribing the BE to COPD patients.

Since the last SR related to the effects of BEs in COPD patients was published, more randomized controlled trials (RCTs) investigating those BEs have become available. These newly available RCTs provide an opportunity, to better identify the effects of BEs in COPD patients. So, the purpose of this study was to update evidence of the effects of BEs on ventilation, exercise capacity, dyspnea, and QoL, in COPD patients.

\section{MATERIALS AND METHODS}

This review was conducted in accordance with the Preferred Reporting Items for Systematic Reviews and MetaAnalyses (PRISMA) [13]. The systematic review protocol was registered in PROSPERO prior synthesis of the result (Registration No. CRD42018102995).

\section{Eligibility criteria}

RCTs investigating the effects of BEs in COPD patients with any stage of severity, were targeted. RCTs with crossover design were also included if the washout period was sufficient for outcomes of interest to return to the baseline, to minimize carry-over effects. In this study, BE was operationally defined as any breathing technique that had a physiological effect on patients. Outcomes of interest included ventilation, exercise capacity, dyspnea, and QoL. RCTs were excluded if (1) they included patients with unstable COPD, on ventilator, or with other diseases, (2) they were published in any language other than English, (3) the BE was less than $50 \%$ of total treatment in the experimental group, (4) they were not the measured 
outcomes of interest, and (5) the control group also received BE.

\section{Search strategy}

Five electronic databases (MEDLINE, CINAHL, Cochrane, Scopus, and ScienceDirect) were searched, from inception of the database to May 10, 2018. Search strategies used for all databases were as follow: ([“COPD” or "Chronic obstructive pulmonary disease" or "Chronic obstructive airway disease" or "Chronic obstructive lung disease"] AND ["Breathing"] AND ["Ventilation" or "Chest physical therapy" or "Chest physiotherapy" or "Pulmonary rehabilitation" or "Respiratory rehabilitation"]).

\section{Study selection}

Two investigators ( $\mathrm{NU}$ and $\mathrm{CK}$ ) independently performed the title, abstract, and full-text search of eligible studies. Accuracy of the search and screen process was confirmed by comparing information obtained during each step of the study collection. In the case of conflicting results, a third investigator (AT) was consulted and participated in the discussion to resolve the concern.

\section{Data extraction}

According to the Cochrane guidelines, the data extraction form was created and applied independently, by two authors (NU and CK) for data extraction. Characteristics of the included studies such as subjects, intervention protocols, and outcome measures, were extracted and compared for consistency, and completeness between investigators. The author (AT) was consulted for final consensus in case of disagreement between the two investigators.

\section{Risk of bias assessment}

Methodological quality of each study was assessed, using the Cochrane Collaboration's tool for assessing risk of bias (The Cochrane Collaboration, London, UK) and classified as low or high risk [14]. Seven criteria of risk of bias including random sequence generation, allocation concealment, selective reporting, blinding of participants and personnel, blinding of outcome assessors, incomplete outcome data, and other bias, were assessed. When risk of bias was low for four or more criteria, the study was rated as low risk of bias. Otherwise, the study was rated as high risk of bias.

\section{Quality of evidence assessment}

The two investigators (NU and $\mathrm{CK}$ ) independently assessed the quality of each outcome, using the Grading of Recommendation Assessment, Development, and Evaluation (GRADE) for rating quality of evidence [15]. Each outcome of interest was classified as high, moderate, low, and very low level of quality of evidence. Quality of evidence of each outcome was downgraded because of the following criteria: limitation of study design ( $>25 \%$ of participants from included studies with high risk of bias); presence of publication bias (asymmetrical funnel plot); indirectness of population, interventions, outcome measures, and comparison of the study; imprecision of measurement ( $<400$ participants); and inconsistency of results $\left(\mathrm{I}^{2} \geq 50 \%\right)$.

\section{Data analysis}

For each outcome, mean change from baseline to postintervention or mean of post-intervention and its standard deviation, were computed and used for metaanalyses (RevMan 5.3, The Cochrane Collaboration). Mean difference (MD) and standardized mean difference (SWD) and its 95\% confidence interval were used to identify overall differences between the treatment and control groups among studies, when data were measure on the same scales. In contrast, SWD was used, when outcomes measured on different scales. Statistic of $\mathrm{I}^{2}$ test was used to identify heterogeneity among studies, within each meta-analysis. When heterogeneity was low $\left(\mathrm{I}^{2}<50 \%\right)$, the fixed-effect model of meta-analysis was used. When heterogeneity was moderate to high $\left(\mathrm{I}^{2} \geq 50 \%\right)$, random-effect of meta-analysis was used [14].

\section{RESULTS}

\section{Study selection}

Fig. 1 presents the screening process, of included studies. Initially, 3,442 articles were identified. After removal of duplication and screening of titles and abstracts, 119 articles were left for full-text screening, and 100 articles were excluded. Finally, 19 studies meeting the criteria were included in this study [16-34]. 


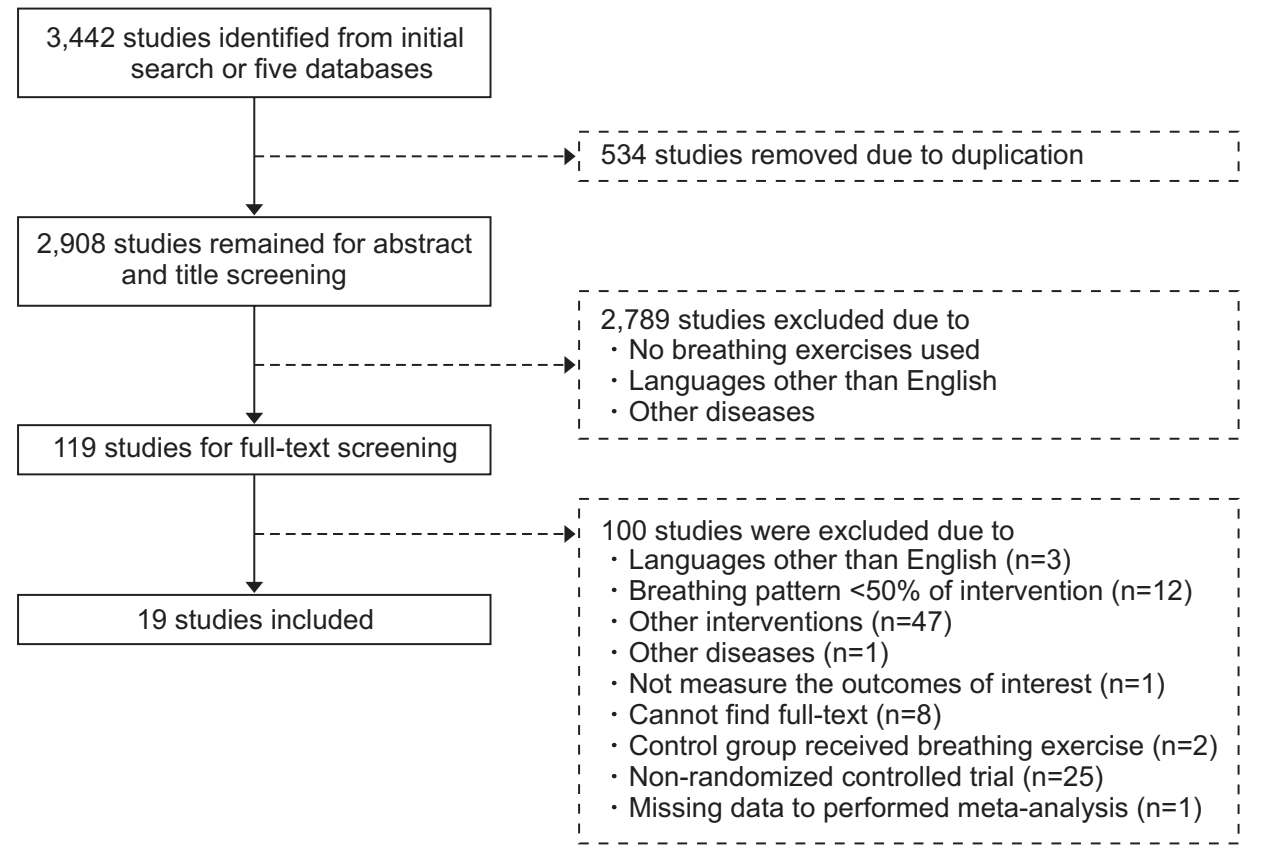

Fig. 1. Flow diagram of included studies selection process.

\section{Study characteristics}

Table 1 summarizes characteristics of the 19 included RCTs. Eight studies were RCTs with cross-over design, with appropriate washout period [17,19-21,24,31-33]. The total number of subjects included in each study ranged from eight to 150 participants, and the total number of subjects included in this study was 745 .

In this study, effects of six types of BE were studied. These BEs were PLB, VF training, VF plus exercise, singing, DBE, and combined BEs (combination of DBE with other BEs). Duration of BE training varied largely among studies, from one session to 24 weeks.

Outcomes of interest of this study were ventilation, dyspnea, exercise capacity, and QoL. Ventilation-related outcomes included respiratory rate (RR), tidal volume $\left(V_{T}\right)$, inspiratory time $\left(T_{i}\right)$, expiratory time $\left(T_{e}\right)$, minute ventilation $\left(\mathrm{V}_{\mathrm{e}}\right)$, total respiratory time $\left(\mathrm{T}_{\text {tot }}\right)$, mean inspiratory flow $\left(\mathrm{V}_{\mathrm{T}} / \mathrm{T}_{\mathrm{i}}\right)$, oxygen saturation $\left(\mathrm{SpO}_{2}\right)$, inspiratory capacity (IC), and control of breathing (breath hold and single breath counting). Dyspnea-related outcome measures were the visual analog scale, Borg scale, modified Borg scale, and Global Rating of Change scale. Exercise capacity-related measures were rate of oxygen consumption $\left(\mathrm{VO}_{2}\right)$, heart rate (HR), 6-minute walk distance (6MWD), and distance of incremental shuttle walk test (ISWT). Last, QoL related outcome measures were the Short Form-36 Health Survey Questionnaire (SF-36),
Hospital and Anxiety Depression scale (HAD), and St. George's Respiratory Questionnaire (SGRQ) score.

\section{Quality assessment}

Table 2 summarizes risk of bias, of included studies. All studies provided sufficient details of random sequence generation, except one study [21]. Only four studies provided adequate detail, of allocation concealment $[26,28,33,34]$. Three studies reported blinding participants and researchers $[20,28,34]$, while other studies had no blinding of participants and researchers. Eight studies reported blinding of outcome assessors [20,26,28$31,33,34]$. Incomplete data were reported in all studies. All studies had low risk for selective reporting bias. Low risk of other biases was found in 14 studies [16$20,22,23,25,27-31,34]$, and insufficient detail was found in five studies $[21,24,26,32,33]$. Based on the seven criteria, 16 of 19 studies were classified as low risk of bias, and the remaining four were high risk of bias. Observational analyses of funnel plots, suggested no publication bias of all outcome measures. Directness of population, interventions, outcome measures, and comparison in each study was observed, and indicated no indirectness in each outcome of meta-analysis.

\section{Analyses of outcome measures}

In the 19 included studies, the effects of PLB, VF train- 


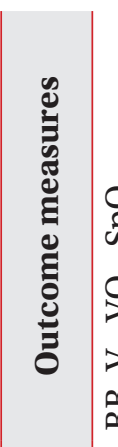

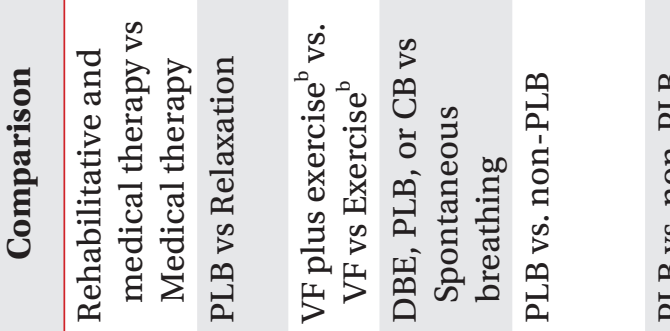

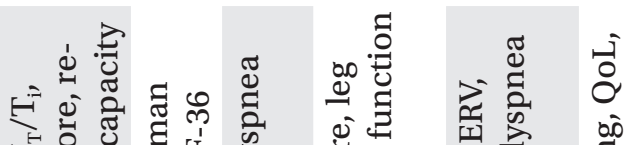
$>>0$ जि की

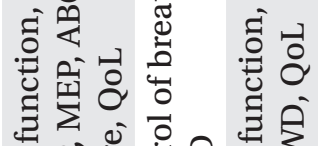

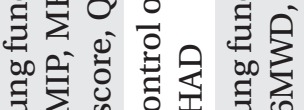
กิ

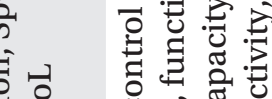
H

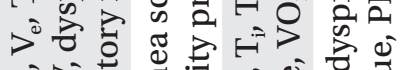

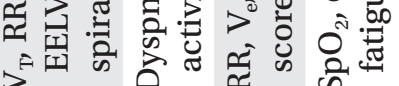

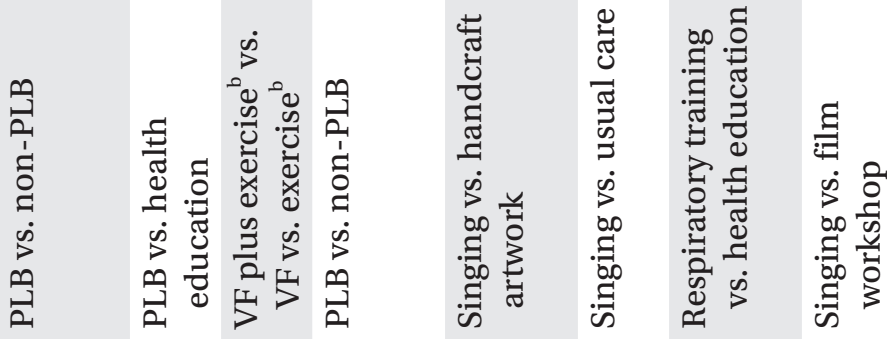
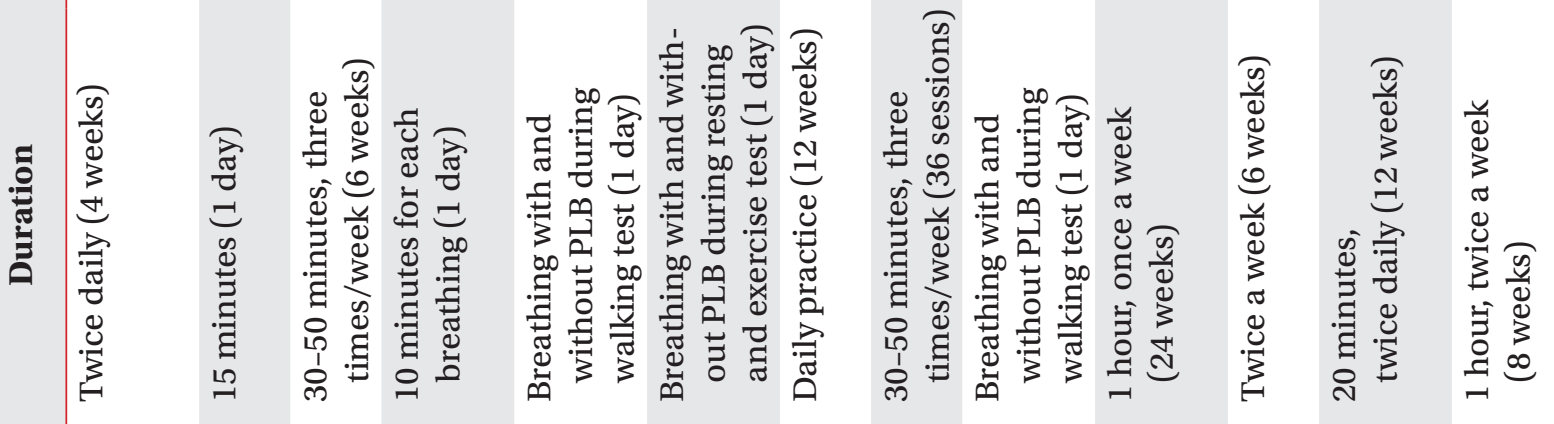

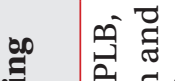

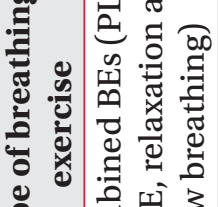

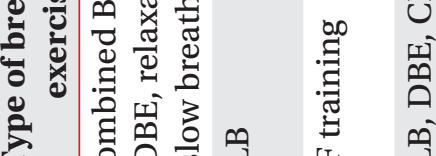

坣

楽
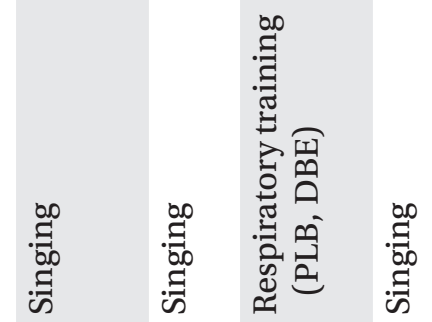

룰

(2)

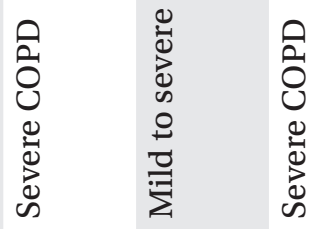
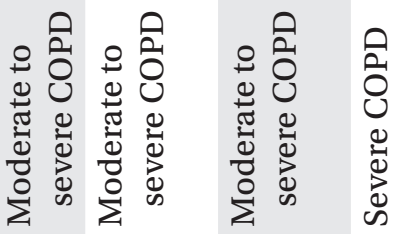

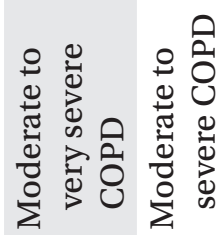

कूँ

ำ

$\stackrel{+}{+}$

$\infty$ 아 है ले

ले

กั ค

太

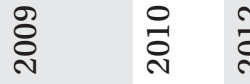

จั้ง 


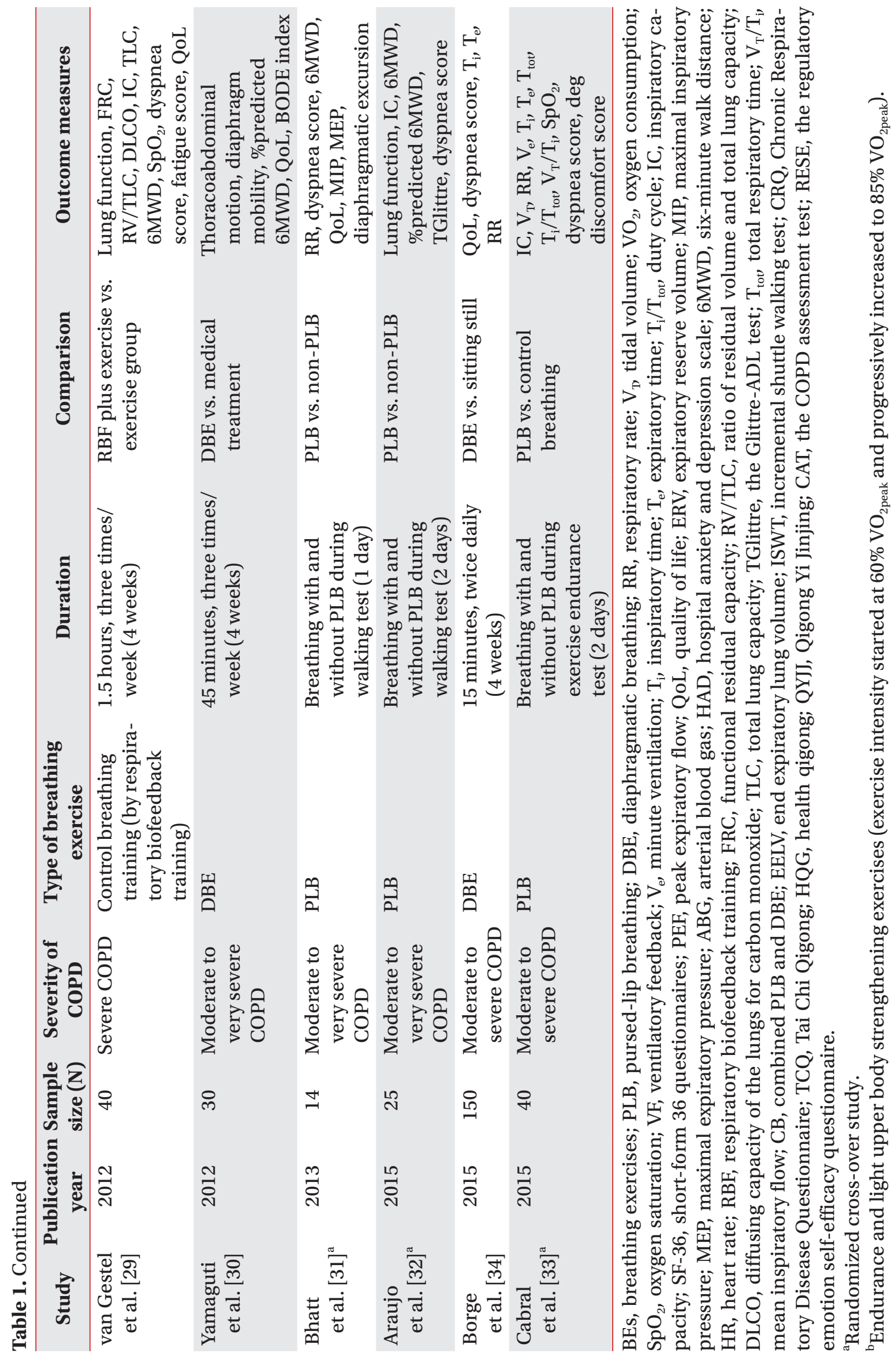




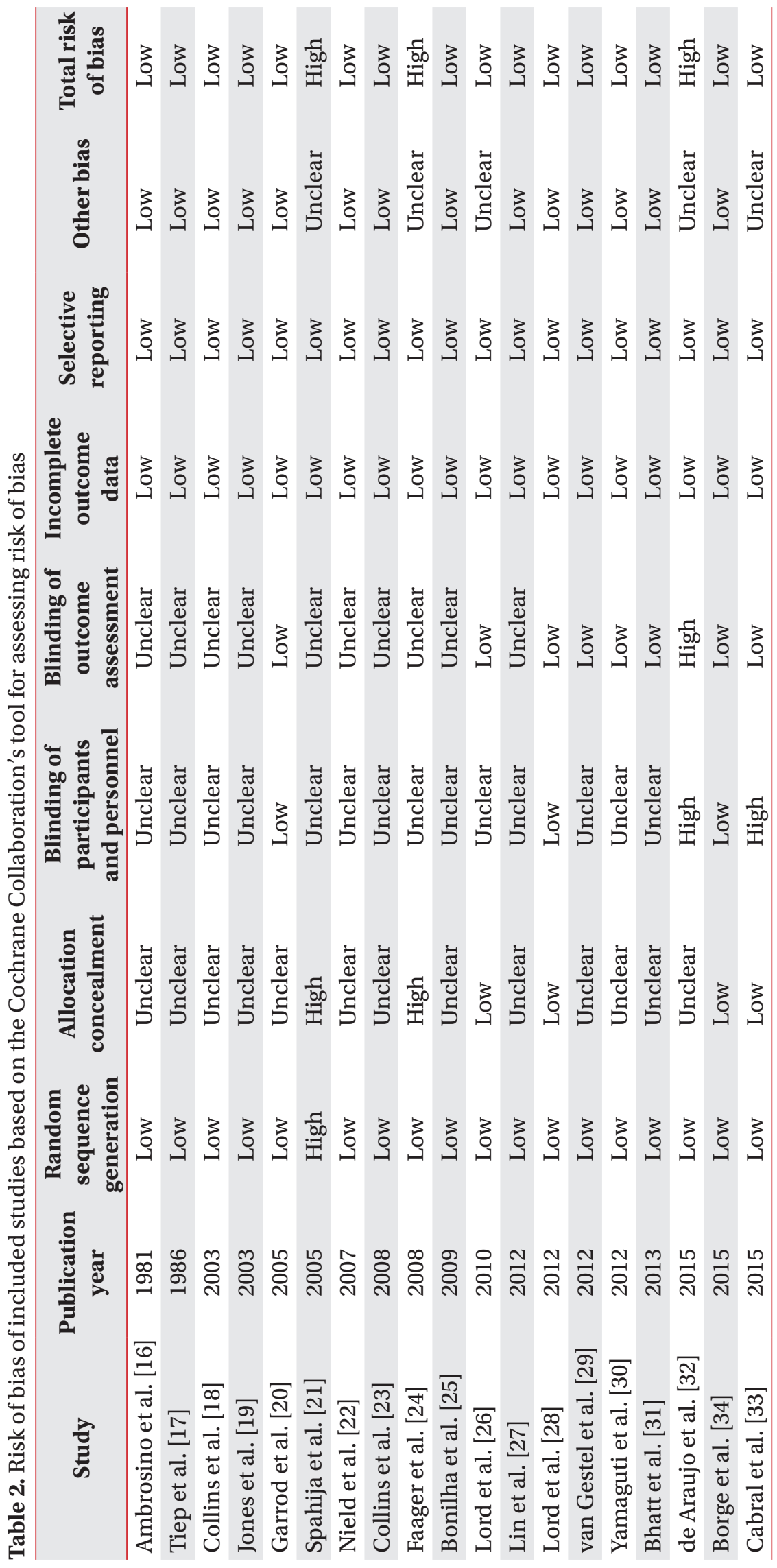


ing alone, VF plus exercise, singing, DBE, and combined BEs were examined. According to the GRADE approach, quality of evidence of PLB (Supplementary Table S1) and VF plus exercise (Supplementary Table S2), was downgraded to low to moderate because of a small sample size, heterogeneity among studies, and/or low methodological quality of included studies. For VF training alone (Supplementary Table S3), singing (Supplementary Table S4), and combined BEs (Supplementary Table S5), the quality of evidence was moderate because of a small sample size. For DBE, quality of evidence was low because of a small sample size and heterogeneity among studies (Supplementary Table S6).

\section{Effects of breathing exercise \\ Pursed-lip breathing}

PLB significantly improved RR ( $\mathrm{p}<0.00001$; Fig. 2) [17,20,21,31,33], $\mathrm{V}_{\mathrm{T}}\left(\mathrm{p}=0.0004\right.$; Fig. 3) [17,21,33], $\mathrm{T}_{\mathrm{i}}$ ( $\mathrm{p}=0.007$; Fig. 4) $[21,33]$, and $\mathrm{T}_{\text {tot }}(\mathrm{p}=0.0004$; Fig. 5) $[21,33]$ than did the control group. Quality of evidence of these outcomes was moderate. There was no significant between-group difference in other ventilatory related outcomes ( $\mathrm{p}=0.10-0.85)$, dyspnea $(\mathrm{p}=0.15)$, and 6MWD $(\mathrm{p}=0.85)$. Table 1 in data supplements summarizes the effects of PLB.

\section{Ventilatory feedback training alone}

$\mathrm{VO}_{2}$ was significantly lower in VF training than the exercise control ( $p=0.02$; Fig. 6) [18,23]. Quality of evidence of this outcome was moderate. No significant differences

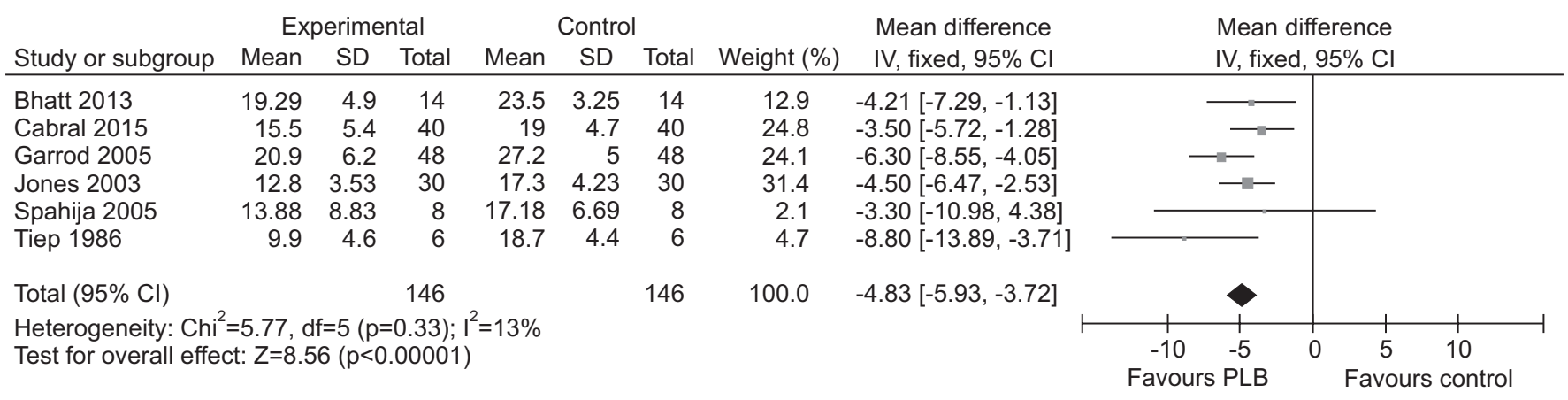

Fig. 2. Forest plot of mean difference in respiratory rate (RR) comparing pursed-lip breathing (PLB) and control.

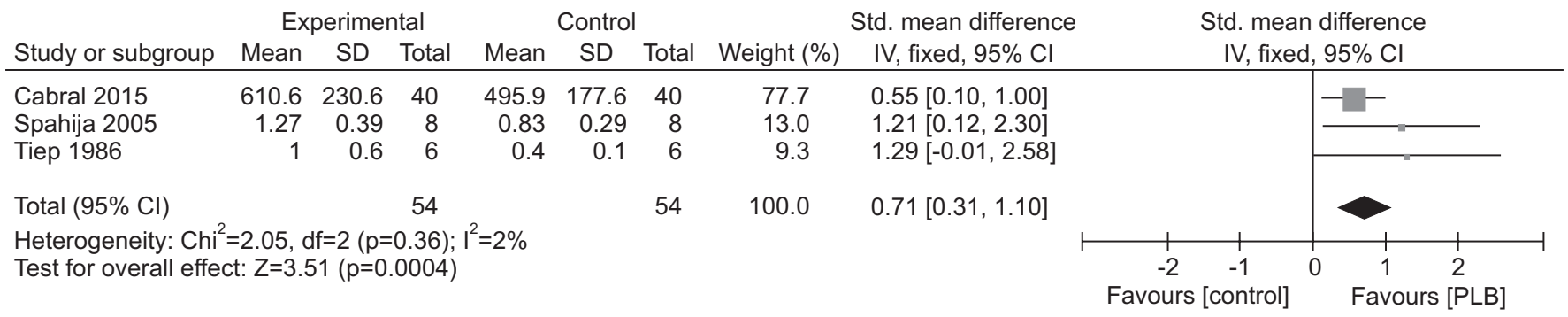

Fig. 3. Forest plot of standard mean difference in tidal volume $\left(\mathrm{V}_{\mathrm{T}}\right)$ comparing pursed lip-breathing (PLB) and control.

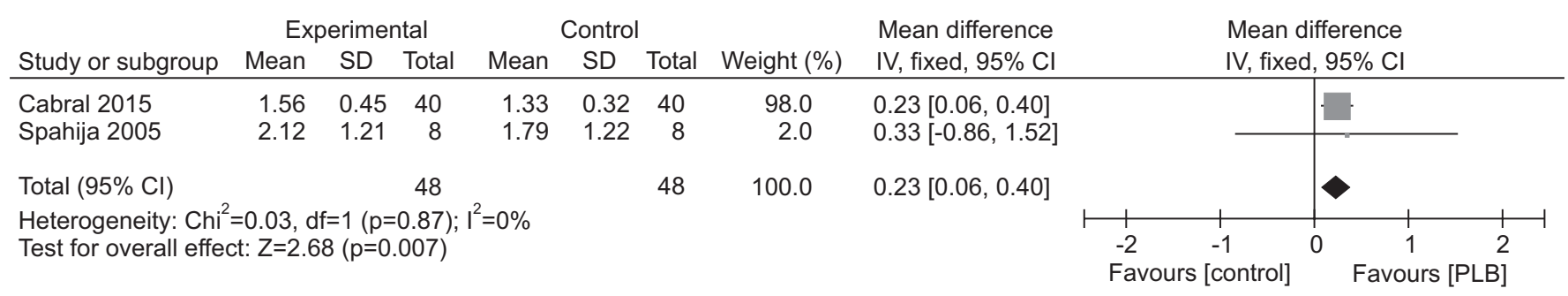

Fig. 4. Forest plot of mean difference in inspiratory time $\left(\mathrm{T}_{\mathrm{i}}\right)$ comparing pursed lip-breathing (PLB) and control. 


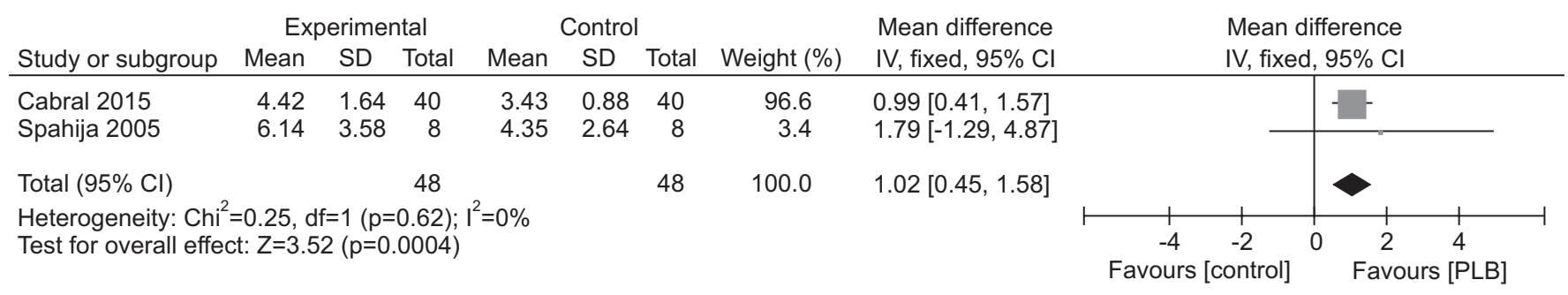

Fig. 5. Forest plot of mean difference in total respiratory time $\left(\mathrm{T}_{\mathrm{tot}}\right)$ comparing pursed lip-breathing (PLB) and control.

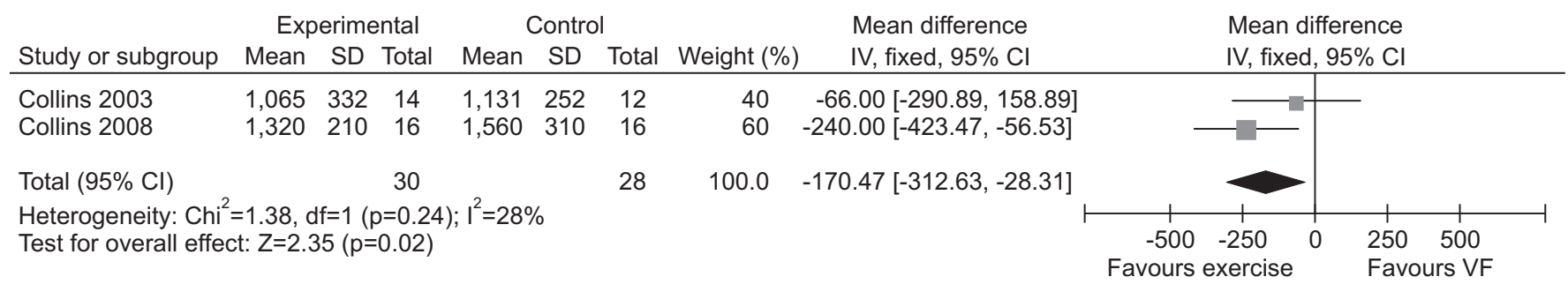

Fig. 6. Forest plot of mean difference in oxygen consumption $\left(\mathrm{VO}_{2}\right)$ comparing ventilatory feedback (VF) and exercise.

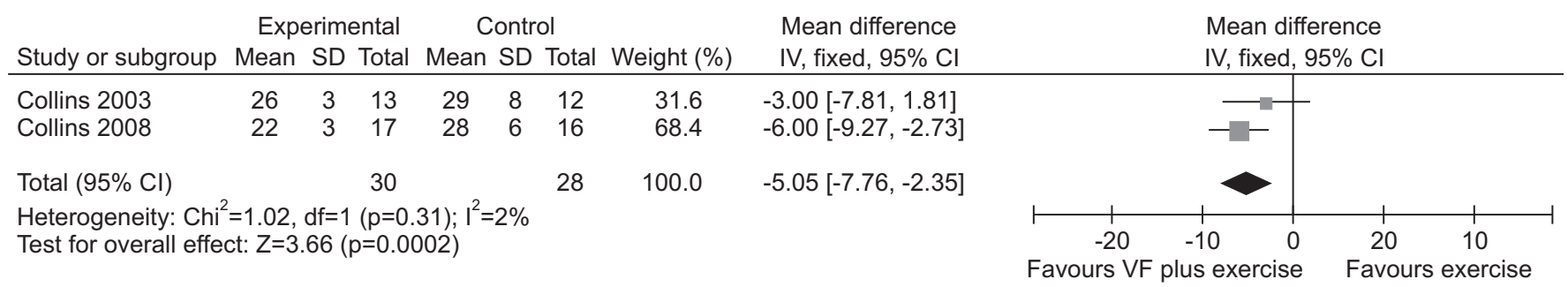

Fig. 7. Forest plot of mean difference in respiratory rate (RR) comparing ventilatory feedback (VF) plus exercise and exercise.

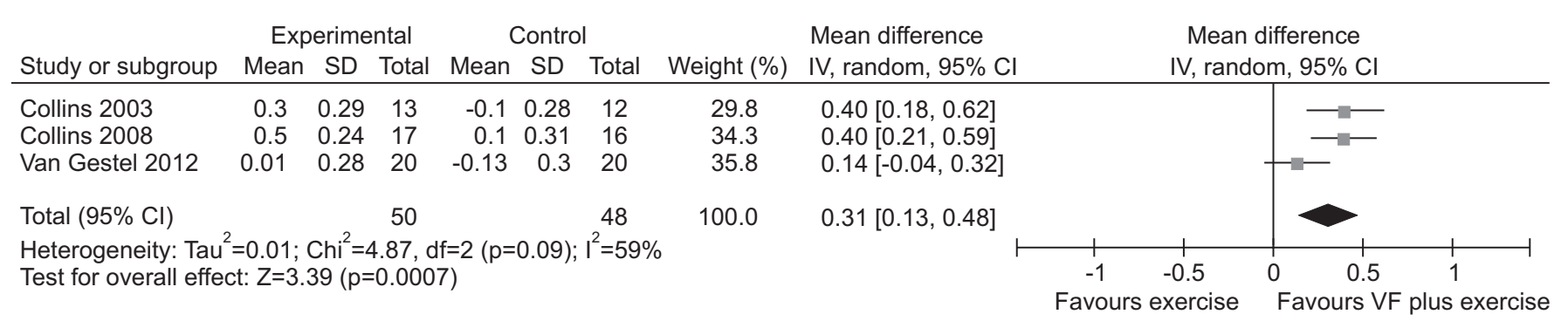

Fig. 8. Forest plot of mean difference in inspiratory capacity (IC) comparing ventilatory feedback (VF) plus exercise and exercise.

in ventilatory related measures $(\mathrm{p}=0.12-0.65)$, dyspnea $(\mathrm{p}=0.15)$, and HR $(\mathrm{p}=0.13)$, were observed between VF training and the control group. The effects of VF training are summarized in data supplements (Supplemetary Table S3).

\section{Ventilatory feedback training plus exercise}

There was moderate quality evidence of a significant improvement in RR in the VF plus exercise group, than the control group ( $\mathrm{P}=0.0002$; Fig. 7) $[18,23]$. Likewise, IC significantly improved in the VF plus exercise group, than the control group ( $p=0.0007$; Fig. 8) $[18,23,29]$. Quality of evidence of this outcome was low. There was no signifi- 
cant between-group difference in other ventilatory related measures $(\mathrm{p}=0.06-0.75)$, dyspnea $(\mathrm{p}=0.83)$, exercise capacity ( $\mathrm{p}=0.68-0.90)$, and QoL ( $\mathrm{p}=0.97)$. The effects of VF plus exercise are summarized in data supplements (Supplemetary Table S2).

\section{Singing exercise}

Breath hold significantly increased in the control group, than the singing group ( $p=0.005$; Fig. 9) $[26,28]$. Quality of evidence of this outcome, was moderate. There was moderate quality evidence of significant difference in the physical component summary of SF-36, between the singing group and the control group ( $\mathrm{p}=0.0005$; Fig. 10) $[26,28]$. However, there was no statistically significant difference in other QoL related measures $(\mathrm{p}=0.07-0.52)$, exercise capacity $(p=0.44)$, and ventilation $(p=0.09)$ between the singing group and the control group. The ef- fects of singing, are summarized in data supplements (Supplemetary Table S4).

\section{Diaphragmatic breathing}

There was low quality evidence of significant improvement in RR, in the DBE group than the control group ( $p=0.05$; Fig. 11) [19,34]. There was no statistically significant difference in dyspnea $(\mathrm{p}=0.47)$ and SGRQ score $(\mathrm{p}=0.58)$, between the DBE group and the control group. Supplemetary Table S6 in data supplements provides a summary of the effects of DBE.

\section{Combined breathing exercises}

RR significantly improved, in the combined BEs group than the control group ( $\mathrm{p}<0.00001$; Fig. 12) $[16,19]$. Quality of evidence for RR, was moderate. There was no significant between-group difference in $\mathrm{SpO}_{2}(\mathrm{p}=0.84)$ and $\mathrm{VO}_{2}$

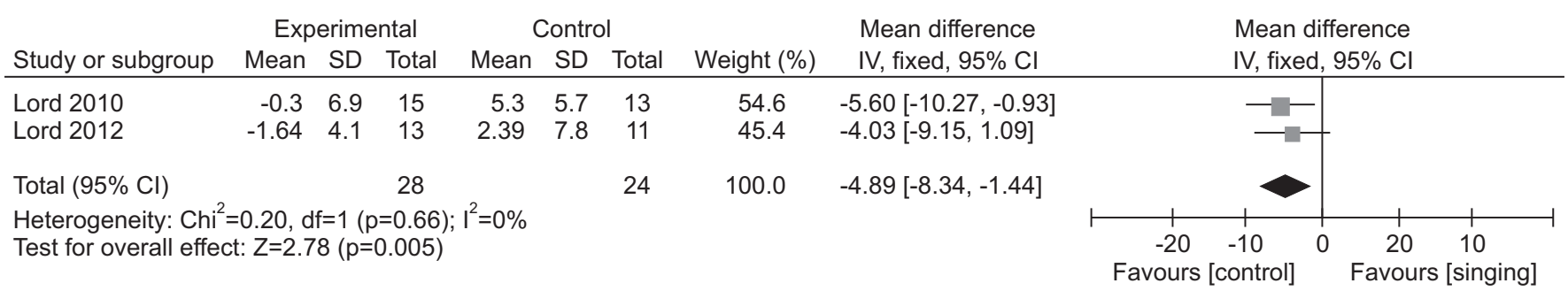

Fig. 9. Forest plot of mean difference in breath hold comparing singing and control.

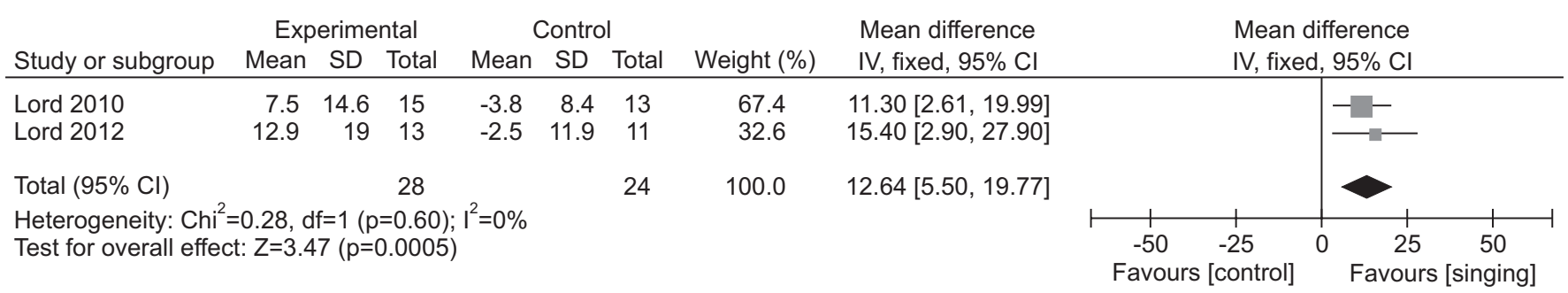

Fig. 10. Forest plot of mean difference in PCS domain of SF-36 comparing singing and control. PCS, physical component summary; SF-36, Short Form-36 Questionnaires.

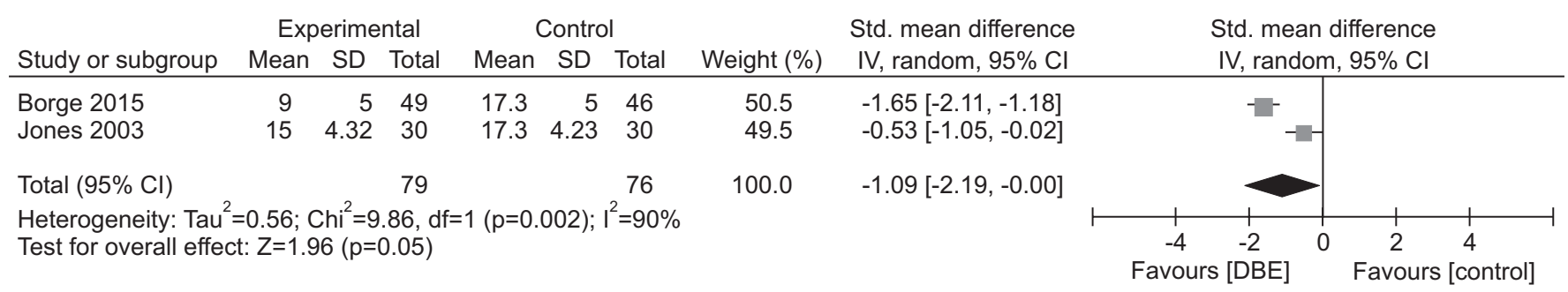

Fig. 11. Forest plot of standard mean difference in respiratory rate (RR) comparing diaphragmatic breathing exercise (DBE) and control. 


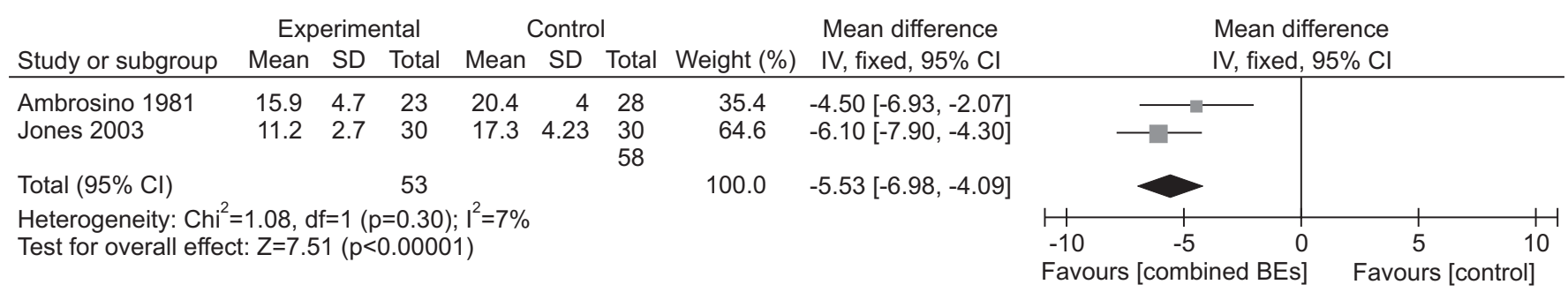

Fig. 12. Forest plot of mean difference in respiratory rate (RR) comparing combined breathing exercises (BEs) and control.

$(\mathrm{p}=0.20)$. The effects of combined BEs are summarized in data supplements (Supplemetary Table S5).

\section{DISCUSSION}

The purpose of this study was to solidify the effects of six types of BE on ventilation, dyspnea, exercise capacity, and QoL in patients with mild to severe COPD. Since the previous SR determining effects of all types of $\mathrm{BE}$ in COPD patients was published in 2012 [9], many studies in this area have been published. However, only four RCTs [31-34] met the criteria of our studies. These studies investigated the effects of PLB [31-33] and DBE [34] in COPD patients. Three RCTs related to PLB provided support relative to the effects of PLB on ventilation-related outcomes [31-33]. The study related to DBE allowed us to perform a meta-analysis, to quantify its effects in COPD patients [34].

When compared to the control groups, PLB, VF plus exercise, DBE, and combined BEs significantly improved several ventilation-related outcomes $\left(R R, V_{t}, T_{i}, I C\right.$, and $\mathrm{T}_{\text {tot }}$ ), with RR the most consistently improved. In contrast, VF training alone was less effective in improving exercise capacity as indicated by a significant decrease in $\mathrm{VO}_{2}$, than the exercise control group. Singing exercise significantly improved the physical component of QoL, but was less effective in improving breath hold time than did the control group. All types of BE studies did not significantly improve sensation of dyspnea, when compared to the control groups. Because of low to moderate quality of evidence and large variability among protocols of the $\mathrm{BE}$ and control groups, recommendation for using the BEs to improve ventilation, exercise capacity, and QoL in patients with mild to very severe COPD is conditional.

\section{Pursed-lip breathing}

PLB has been shown to improve, several ventilation related outcomes. When compared to the control group, PLB was found to positively impact RR, $V_{T}, T_{i}$, and $T_{\text {tot }}$. Results were consistent, with those previously reported in COPD patients $[8,9,11]$. Also, our results indicate positive effects of PLB on $T_{i}$ and $T_{\text {tot }}$ with decrease in RR and increase in $\mathrm{V}_{\mathrm{T}}$. Reduction in $\mathrm{RR}$ with increase in $\mathrm{V}_{\mathrm{T}}$ suggests reduction in airway collapse, airway resistance, and air trapping in the lungs [35]. These changes contribute to reduction of the mechanical load, imposed on respiratory muscles [35-37] and eventually dyspnea [35,36]. Based on the result of meta-analysis, PLB is more effective in improving ventilation, than the control group.

PLB is not superior to the control group on improving sensation of dyspnea in COPD patients. It is possible that the duration of PLB training in the included studies was not sufficient to positively impact sensation of dyspnea in COPD patients. Holland et al. [9] reported significant decrease in sensation of dyspnea, after 8 weeks of PLB training. However, three of four included studies used a very short training period of 1 day [20,31] to 2 days [33]. Only one study used a 12-week training period of PLB [22]. So, further studies are needed to identify the effects of the length of PLB training on sensation of dyspnea in COPD patients.

\section{Ventilatory feedback training and ventilatory feedback training plus exercise}

This study indicated that VF plus exercise showed positive impact on ventilation (RR and IC), whereas VF alone was less effective in improving exercise capacity than the control group $[28,23]$. Results were consistent with the previous study, wherein the exercise group tended to improve exercise capacity as compared to VF training [9]. Differences in training protocols between the VF and VF plus exercise groups, may have an influence on outcomes. These two groups received similar VF training protocol, but different exercise protocols. In the VF train- 
ing group, patients performed light exercise training to become familiar with use of breathing during activities of daily living (ADLs). Neither load nor exercise progression was used in the VF training group $[18,23]$. For VF plus exercise, patients performed progressive exercise training with intensity at $60 \%-85 \%$ of peak $\mathrm{VO}_{2}$ peak for 25-45 minutes [18,23]. Exercise protocol was also used in the control group [18,23], resulting in no significant difference in exercise capacity in the VF plus exercise group and the control group. Based on the low to moderate quality of evidence, VF training plus exercise and progressive exercise training should be integrated into a pulmonary rehabilitation program to improve ventilation and exercise capacity of COPD patients.

\section{Singing exercise}

Singing exercise showed a positive effect on the physical component of SF-36 as compared to the control group. This result is consistent with that of the previous study, wherein reduction in air trapping and better performance of physical activity and ADLs were observed in COPD patients [26]. However, improvement of mental status such as anxiety and depression symptoms noted in the singing group was similar to that of the control group. It is possible that the handicraft artwork or film workshop used in the control group also had positive psychosocial effects such as increased patient's sociability $[25,28]$, similar to those observed in the singing group. As a result, there was no significant difference in mental status between the singing group and control group.

The singing exercise was less effective in improving breath hold time than the control group. This unexpected result may be attributable to use of controlled or comfortable breathing pattern during the singing exercise. As a result, lower inspiration was noted in the singing exercise as compared to quiet breathing $[26,28]$. Although the singing exercise has been shown to have a positive effect on improving function of respiratory muscles and control of breathing [26], insufficient training sessions and/ or incompliance with the exercise program may limit effectiveness on improving breath hold time in COPD patients. Also, the breath hold test may not be an appropriate measure in COPD patients, as it has been shown to induce sensation of dyspnea usually experienced by COPD patients. In contrast, it is more appropriate, for evaluating patients with hyperventilation [10]. Based on the moderate quality of evidence, the singing exercise may be potentially used to improve the physical component of QoL, but not breath hold time in COPD patients.

\section{Diaphragmatic breathing and combined breathing exercises}

DBE and combined BEs demonstrated significant improvement in RR, compared to the control groups. Combined BEs consisted of DBE, PLB [19], slow breathing, and relaxation techniques [16]. Results of this review are consistent with those previously reported, wherein RR was significantly reduced with DBE $[19,34]$ or combined DBE with PLB [19]. Reduction of RR was associated with lower $\mathrm{VO}_{2}$ [19], and less sympathetic activity [34]. These two effects may lead to reduction in sensation of dyspnea in COPD patients. However, sensation of dyspnea and QoL were not significantly improved in the DBE group compared to the control group. Although the previous study revealed that there was improvement of dyspnea and QoL after four weeks of DBE training [30], an inconsistency in results of DBE on dyspnea and QoL among previous studies, also exists $[9,12]$. DBE was also reported to induce dyspnea by increasing the afford of inspiratory muscles and incoordinate chest wall motion [12]. Thus, the effect of DBE on dyspnea remains inconclusive. Further studies should focus on the effects of DBE on dyspnea and QoL in COPD patients. Based on low to moderate quality of evidence, DBE and combined BEs may be useful for improvement of ventilation in COPD patients.

\section{Limitations}

Although this study added more RCTs to the metaanalysis to solidify effects of BEs, a few limitations persist in this study. First, relevant articles were electronically searched, and no manual search was performed. It is possible that a few more relevant articles may not be included in this study. However, when compared to previous systematic reviews $[9,11]$, no discrepancy in studies included in our and previous studies was observed. This result suggests minor to no effect relative to manual search of literature on the outcomes of this study. Second, only RCTs published in English language were included in this study. However, previous studies reported that language bias had minimal effect on the result of meta-analysis [38]. Third, patients with mild to severe COPD were included, and the majority of patients 
had moderate to very severe COPD. Variation in patients' severity of stage of COPD may increase heterogeneity of results, leading to downgrading of the quality of evidence, and some of the non-significant results observed. It is possible that differences in patient's severity or stage of COPD may influence the effects of the BEs on these outcomes. A separate analysis of these outcomes based on the stage of COPD may provide different results compared to our study. However, the studies currently available commonly included COPD patients with a range of severity, as compared to one specific stage of severity. Further studies with a larger sample size and specific severity of COPD patients will minimize these limitations. Additionally, although many RCTs were included in our meta-analysis, large variation in the protocol of BEs and outcome measures were observed, leading to heterogeneity of results. Further studies with a more uniform treatment protocol and outcome measures, and a larger sample size will be needed, to confirm the effects of BEs in COPD patients.

\section{Conclusion}

Based on low to moderate quality of evidence, PLB, VF plus exercise, DBE, and combined BEs, are conditionally recommended to improve ventilation. Singing exercise can also be used to improve physical component of QoL in COPD patients. However, all types of BEs do not significantly improve sensation of dyspnea relative to the control group. Since the effects of BEs exercise vary significantly between types of $\mathrm{BE}$ as well as outcome measures, care must be practiced when selecting BEs to ensure its effectiveness specific to COPD patients.

\section{CONFLICT OF INTEREST}

No potential conflict of interest relevant to this article was reported.

\section{ACKNOWLEDGMENTS}

This study was supported by a scholarship from "The 100th Anniversary Chulalongkorn University Fund for Doctoral Scholarship" and also "The 90th Anniversary Chulalongkorn University Fund (Ratchadaphiseksomphot Endowment Fund), and supported in part by The Thailand Research Fund (No. MRG-5980084).

\section{AUTHOR CONTRIBUTION}

Conceptualization: Ubolnuar N, Tantisuwat A, Mathiyakom W. Methodology: Ubolnuar N, Kruapanich C, Tantisuwat A. Formal analysis: Ubolnuar N, Lertmaharit S. Funding acquisition: Ubolnuar N, Tantisuwat A. Project administration: Tantisuwat A. Writing - original draft: Ubolnuar N. Writing - review and editing: Ubolnuar N, Tantisuwat A, Mathiyakom W, Thaveeratitham P. Approval of final manuscript: all authors.

\section{SUPPLEMENTARY MATERIALS}

Supplementary materials can be found via https://doi. org/10.5535/arm.2019.43.4.509. Table S1. Summary of the effects of PLB compared to control group. Table S2. Summary of the effects of VF plus exercise compared to control group. Table S3. Summary of the effects of VF training compared to control group. Table S4. Summary of the effects of singing compared to control group. Table S5. Summary of the effects of combined BEs compared to control group. Table S6. Summary of the effects of DBE compared to control group.

\section{REFERENCES}

1. World Health Organization. Chronic respiratory disease: burden of COPD [Internet]. Geneva, Switzerland: World Health Organization; c2019 [cited at 2019 Jul 1]. Available from: https://www.who.int/respiratory/copd/burden/en/.

2. Watchie J. Cardiovascular and pulmonary physical therapy: a clinical manual. 2nd ed. London: Elsevier Health Sciences; 2010.

3. Global Initiative for Chronic Obstructive Lung Disease. Global strategy for the diagnosis, management and prevention of chronic obstructive pulmonary disease (update 2015). Fontana, WI: Global Initiative for Chronic Obstructive Lung Disease; 2015.

4. Wouters EF. Chronic obstructive pulmonary disease. 5 . Systemic effects of COPD. Thorax 2002;57:1067-70.

5. Berry MJ, Rejeski WJ, Miller ME, Adair NE, Lang W, Foy CG, et al. A lifestyle activity intervention in patients with chronic obstructive pulmonary disease. Respir Med 2010;104:829-39.

6. World Health Organization. Chronic respiratory dis- 
ease: COPD management [Internet]. Geneva, Switzerland: World Health Organization; c2019 [cited at 2019 Jul 1]. Available from: https://www.who.int/respiratory/copd/management/en/.

7. Gosselink R. Controlled breathing and dyspnea in patients with chronic obstructive pulmonary disease (COPD). J Rehabil Res Dev 2003;40(5 Suppl 2):25-33.

8. Roberts SE, Stern M, Schreuder FM, Watson T. The use of pursed lips breathing in stable chronic obstructive pulmonary disease: a systematic review of the evidence. Phys Ther Rev 2009;14(4):240-6.

9. Holland AE, Hill CJ, Jones AY, McDonald CF. Breathing exercises for chronic obstructive pulmonary disease. Cochrane Database Syst Rev 2012;10:CD008250.

10. Lewis A, Cave P, Stern M, Welch L, Taylor K, Russell J, et al. Singing for lung health: a systematic review of the literature and consensus statement. NPJ Prim Care Respir Med 2016;26:16080.

11. Mayer AF, Karloh M, Dos Santos K, de Araujo CL, Gulart AA. Effects of acute use of pursed-lips breathing during exercise in patients with COPD: a systematic review and meta-analysis. Physiotherapy 2018;104:917.

12. Cahalin LP, Braga M, Matsuo Y, Hernandez ED. Efficacy of diaphragmatic breathing in persons with chronic obstructive pulmonary disease: a review of the literature. J Cardiopulm Rehabil 2002;22:7-21.

13. Moher D, Liberati A, Tetzlaff J, Altman DG; PRISMA Group. Preferred reporting items for systematic reviews and meta-analyses: the PRISMA statement. Ann Intern Med 2009;151:264-9, W64.

14. Higgins JP, Green S. Cochrane handbook for systematic reviews of interventions (Version 5.1.0) [Internet]. London: The Cochrane Collaboration; 2011 [cited 2019 Jul 1]. Available from: https://handbook-5-1.cochrane.org/.

15. Balshem H, Helfand M, Schunemann HJ, Oxman AD, Kunz R, Brozek J, et al. GRADE guidelines. 3. Rating the quality of evidence. J Clin Epidemiol 2011;64:4016.

16. Ambrosino N, Paggiaro PL, Macchi M, Filieri M, Toma G, Lombardi FA, et al. A study of short-term effect of rehabilitative therapy in chronic obstructive pulmonary disease. Respiration 1981;41:40-4.

17. Tiep BL, Burns M, Kao D, Madison R, Herrera J. Pursed lips breathing training using ear oximetry. Chest 1986;
90:218-21.

18. Collins EG, Fehr L, Bammert C, O'Connell S, Laghi F, Hanson $\mathrm{K}$, et al. Effect of ventilation-feedback training on endurance and perceived breathlessness during constant work-rate leg-cycle exercise in patients with COPD. J Rehabil Res Dev 2003;40(5 Suppl 2):35-44.

19. Jones AY, Dean E, Chow CC. Comparison of the oxygen cost of breathing exercises and spontaneous breathing in patients with stable chronic obstructive pulmonary disease. Phys Ther 2003;83:424-31.

20. Garrod R, Dallimore K, Cook J, Davies V, Quade K. An evaluation of the acute impact of pursed lips breathing on walking distance in nonspontaneous pursed lips breathing chronic obstructive pulmonary disease patients. Chron Respir Dis 2005;2:67-72.

21. Spahija J, de Marchie M, Grassino A. Effects of imposed pursed-lips breathing on respiratory mechanics and dyspnea at rest and during exercise in COPD. Chest 2005;128:640-50.

22. Nield MA, Soo Hoo GW, Roper JM, Santiago S. Efficacy of pursed-lips breathing: a breathing pattern retraining strategy for dyspnea reduction. J Cardiopulm Rehabil Prev 2007;27:237-44.

23. Collins EG, Langbein WE, Fehr L, O'Connell S, Jelinek C, Hagarty E, et al. Can ventilation-feedback training augment exercise tolerance in patients with chronic obstructive pulmonary disease? Am J Respir Crit Care Med 2008;177:844-52.

24. Faager G, Stâhle A, Larsen FF. Influence of spontaneous pursed lips breathing on walking endurance and oxygen saturation in patients with moderate to severe chronic obstructive pulmonary disease. Clin Rehabil 2008;22:675-83.

25. Bonilha AG, Onofre F, Vieira ML, Prado MY, Martinez JA. Effects of singing classes on pulmonary function and quality of life of COPD patients. Int J Chron Obstruct Pulmon Dis 2009;4:1-8.

26. Lord VM, Cave P, Hume VJ, Flude EJ, Evans A, Kelly $\mathrm{JL}$, et al. Singing teaching as a therapy for chronic respiratory disease: a randomized controlled trial and qualitative evaluation. BMC Pulm Med 2010;10:41.

27. Lin WC, Yuan SC, Chien JY, Weng SC, Chou MC, Kuo HW. The effects of respiratory training for chronic obstructive pulmonary disease patients: a randomised clinical trial. J Clin Nurs 2012;21:2870-8.

28. Lord VM, Hume VJ, Kelly JL, Cave P, Silver J, Waldman 
M, et al. Singing classes for chronic obstructive pulmonary disease: a randomized controlled trial. BMC Pulm Med 2012;12:69.

29. van Gestel AJ, Kohler M, Steier J, Teschler S, Russi EW, Teschler H. The effects of controlled breathing during pulmonary rehabilitation in patients with COPD. Respiration 2012;83:115-24.

30. Yamaguti WP, Claudino RC, Neto AP, Chammas MC, Gomes AC, Salge JM, et al. Diaphragmatic breathing training program improves abdominal motion during natural breathing in patients with chronic obstructive pulmonary disease: a randomized controlled trial. Arch Phys Med Rehabil 2012;93:571-7.

31. Bhatt SP, Luqman-Arafath TK, Gupta AK, Mohan A, Stoltzfus JC, Dey T, et al. Volitional pursed lips breathing in patients with stable chronic obstructive pulmonary disease improves exercise capacity. Chron Respir Dis 2013;10:5-10.

32. de Araujo CL, Karloh M, Dos Reis CM, Palu M, Mayer AF. Pursed-lips breathing reduces dynamic hyperinflation induced by activities of daily living test in patients with chronic obstructive pulmonary disease: a randomized cross-over study. J Rehabil Med 2015;47:957-62.
33. Cabral LF, D'Elia Tda C, Marins Dde S, Zin WA, Guimaraes FS. Pursed lip breathing improves exercise tolerance in COPD: a randomized crossover study. Eur J Phys Rehabil Med 2015;51:79-88.

34. Borge CR, Mengshoel AM, Omenaas E, Moum T, Ekman I, Lein MP, et al. Effects of guided deep breathing on breathlessness and the breathing pattern in chronic obstructive pulmonary disease: a doubleblind randomized control study. Patient Educ Couns 2015;98:182-90.

35. Kant S, Singh GV. Breathing exercises as adjuvant in the management of COPD: an overview. Lung India 2006;23(4):165-9.

36. Gigliotti F, Coli C, Bianchi R, Romagnoli I, Lanini B, Binazzi B, er al. Exercise training improves exertional dyspnea in patients with COPD: evidence of the role of mechanical factors. Chest 2003;123:1794-802.

37. Rabe KF. Improving dyspnea in chronic obstructive pulmonary disease: optimal treatment strategies. Proc Am Thorac Soc 2006;3:270-5.

38. Juni P, Holenstein F, Sterne J, Bartlett C, Egger M. Direction and impact of language bias in meta-analyses of controlled trials: empirical study. Int J Epidemiol 2002;31:115-23. 


\section{SUPPLEMENTARY MATERIALS}

Table S1. Summary of the effects of PLB compared to control group

\begin{tabular}{|c|c|c|c|c|c|}
\hline \multirow{2}{*}{ Outcome } & \multirow{2}{*}{$\begin{array}{c}\text { No. of participants } \\
\text { (studies) }\end{array}$} & \multirow{2}{*}{$\begin{array}{l}\text { Quality of } \\
\text { evidence }\end{array}$} & \multicolumn{2}{|c|}{ Effect estimate (95\% CI) } & \multirow{2}{*}{ p-value } \\
\hline & & & MD & SMD & \\
\hline \multicolumn{6}{|l|}{ Ventilation } \\
\hline $\mathrm{RR}(\mathrm{bpm})$ & $292(6)$ & $\oplus \oplus \oplus \Theta^{\mathrm{a}}$ Moderate & $-4.83(-5.93,-3.72)$ & & $<0.00001^{*}$ \\
\hline $\mathrm{SpO}_{2}(\%)$ & $252(4)$ & $\oplus \oplus \Theta^{\mathrm{a}} \Theta^{\mathrm{b}}$ Low & $1.19(-0.22,2.59)$ & & 0.10 \\
\hline $\mathrm{V}_{\mathrm{T}}(\mathrm{L})$ & $108(3)$ & $\oplus \oplus \oplus \Theta^{\mathrm{a}}$ Moderate & & $0.71(0.31,1.10)$ & $0.0004^{*}$ \\
\hline $\mathrm{V}_{\mathrm{e}}(\mathrm{L} / \mathrm{min})$ & $108(3)$ & $\oplus \oplus \oplus \Theta^{\mathrm{a}}$ Moderate & $0.13(-1.20,1.46)$ & & 0.85 \\
\hline $\mathrm{T}_{\mathrm{i}}(\mathrm{s})$ & $96(2)$ & $\oplus \oplus \oplus \ominus^{\mathrm{a}}$ Moderate & $0.23(0.06,0.40)$ & & $0.007^{*}$ \\
\hline $\mathrm{T}_{\text {tot }}(\mathrm{s})$ & $96(2)$ & $\oplus \oplus \oplus \ominus^{\mathrm{a}}$ Moderate & $1.02(0.45,1.58)$ & & $0.0004^{*}$ \\
\hline $\mathrm{V}_{\mathrm{T}} / \mathrm{T}_{\mathrm{i}}$ & $96(2)$ & $\oplus \oplus \oplus \ominus^{\mathrm{a}}$ Moderate & & $0.28(-0.12,0.69)$ & 0.17 \\
\hline IC & $130(2)$ & $\oplus \oplus \Theta^{\mathrm{a}} \Theta^{\mathrm{c}}$ Low & & $0.11(-0.24,0.45)$ & 0.54 \\
\hline \multicolumn{6}{|c|}{ Sensation of dyspnea } \\
\hline Dyspnea & $284(4)$ & $\oplus \oplus \ominus^{\mathrm{a}} \Theta^{\mathrm{b}}$ Low & & $-1.13(-2.67,0.41)$ & 0.15 \\
\hline \multicolumn{6}{|c|}{ Exercise capacity } \\
\hline $6 \mathrm{MWD}(\mathrm{m})$ & $78(2)$ & $\oplus \oplus \ominus^{\mathrm{a}} \Theta^{\mathrm{c}}$ Low & $3.13(-28.61,34.87)$ & & 0.85 \\
\hline
\end{tabular}

$\mathrm{MD}$, mean difference; SMD, standard mean difference; PLB, pursed lip breathing; RR, respiratory rate; $\mathrm{SpO}_{2}$, oxygen saturation; $\mathrm{V}_{\mathrm{T}}$, tidal volume; $\mathrm{V}_{\mathrm{e}}$, minute ventilation; $\mathrm{T}_{\mathrm{i}}$, inspiratory time; $\mathrm{T}_{\mathrm{to}}$, total respiratory time; $\mathrm{V}_{\mathrm{T}} / \mathrm{T}_{\mathrm{i}}$, mean inspiratory flow; IC, inspiratory capacity; 6MWD, 6-minute walk distance.

${ }^{a}$ Serious imprecision of measurements (sample size $<400$ participants).

${ }^{\mathrm{b}}$ Serious inconsistency of results $\left(\mathrm{I}^{2} \geq 50 \%\right)$.

'Serious risk of bias ( $>25 \%$ of participants from study with high risk of bias).

${ }^{*} \mathrm{p}<0.001$. 
Table S2. Summary of the effects of VF plus exercise compared to control group

\begin{tabular}{|c|c|c|c|c|c|}
\hline \multirow{2}{*}{ Outcome } & \multirow{2}{*}{$\begin{array}{l}\text { No. of participants } \\
\text { (studies) }\end{array}$} & \multirow{2}{*}{$\begin{array}{l}\text { Quality of } \\
\text { evidence }\end{array}$} & \multicolumn{2}{|c|}{ Effect estimate (95\% CI) } & \multirow{2}{*}{ p-value } \\
\hline & & & MD & SMD & \\
\hline \multicolumn{6}{|l|}{ Ventilation } \\
\hline $\mathrm{RR}(\mathrm{bpm})$ & $58(2)$ & $\oplus \oplus \oplus \Theta^{\mathrm{a}}$ Moderate & $-5.05(-7.76,-2.35)$ & & $0.0002^{*}$ \\
\hline $\mathrm{SpO}_{2}(\%)$ & $65(2)$ & $\oplus \oplus \Theta^{\mathrm{a}} \Theta^{\mathrm{b}}$ Low & $0.55(-0.99,2.09)$ & & 0.48 \\
\hline $\mathrm{V}_{\mathrm{T}}(\mathrm{L})$ & $58(2)$ & $\oplus \oplus \oplus \ominus^{\mathrm{a}}$ Moderate & $0.21(-0.01,0.43)$ & & 0.06 \\
\hline $\mathrm{V}_{\mathrm{e}}(\mathrm{L} / \mathrm{min})$ & $58(2)$ & $\oplus \oplus \oplus^{\mathrm{a}} \Theta^{\mathrm{b}}$ Low & $-1.75(-12.57,9.06)$ & & 0.75 \\
\hline $\mathrm{T}_{\mathrm{i}}(\mathrm{s})$ & $58(2)$ & $\oplus \oplus \oplus \ominus^{\text {a }}$ Moderate & $0.07(-0.02,0.17)$ & & 0.14 \\
\hline $\mathrm{T}_{\mathrm{e}}(\mathrm{s})$ & $58(2)$ & $\oplus \oplus \Theta^{\mathrm{a}} \Theta^{\mathrm{b}}$ Low & $0.27(-0.05,0.59)$ & & 0.10 \\
\hline IC (L) & $98(3)$ & $\oplus \oplus \Theta^{\mathrm{a}} \Theta^{\mathrm{c}}$ Low & $0.31(0.13,0.48)$ & & $0.0007^{*}$ \\
\hline \multicolumn{6}{|c|}{ Sensation of dyspnea } \\
\hline Dyspnea & $98(3)$ & $\oplus \oplus \Theta^{\mathrm{a}} \Theta^{\mathrm{b}}$ Low & & $0.08(-0.63,0.79)$ & 0.83 \\
\hline \multicolumn{6}{|l|}{ Exercise capacity } \\
\hline HR (bpm) & $58(2)$ & $\oplus \oplus \oplus \Theta^{\mathrm{a}}$ Moderate & $-1.72(-9.78,6.34)$ & & 0.68 \\
\hline $\mathrm{VO}_{2}(\mathrm{~mL} / \mathrm{min})$ & $58(2)$ & $\oplus \oplus \Theta^{\mathrm{a}} \Theta^{\mathrm{b}}$ Low & $-15.77(-273.84,242.29)$ & & 0.90 \\
\hline \multicolumn{6}{|l|}{ QoL } \\
\hline QoL-CRQ score & $73(2)$ & $\oplus \oplus \Theta^{\mathrm{a}} \Theta^{\mathrm{b}}$ Low & & $0.01(-0.67,0.69)$ & 0.97 \\
\hline
\end{tabular}

$\mathrm{MD}$, mean difference; SMD, standard mean difference; $\mathrm{VF}$, ventilatory feedback; $\mathrm{RR}$, respiratory rate; $\mathrm{SpO}_{2}$, oxygen saturation; $\mathrm{V}_{\mathrm{T}}$, tidal volume; $\mathrm{V}_{\mathrm{e}}$, minute ventilation; $\mathrm{T}_{\mathrm{i}}$, inspiratory time; $\mathrm{T}_{\mathrm{e}}$, expiratory time; IC, inspiratory capacity; $\mathrm{HR}$, heart rate, $\mathrm{VO}_{2}$, oxygen consumption; QoL, quality of life; CRQ, chronic respiratory questionnaires.

${ }^{a}$ Serious imprecision of measurements (sample size $<400$ participants).

${ }^{\mathrm{b}}$ Serious inconsistency of results $\left(\mathrm{I}^{2} \geq 50 \%\right)$.

${ }^{\mathrm{c}}$ Serious risk of bias ( $>25 \%$ of participants from study with high risk of bias).

${ }^{*} \mathrm{p}<0.001$.

Table S3. Summary of the effects of VF training compared to control group

\begin{tabular}{|c|c|c|c|c|c|}
\hline \multirow{2}{*}{ Outcome } & \multirow{2}{*}{$\begin{array}{c}\text { No. of participants } \\
\text { (studies) }\end{array}$} & \multirow{2}{*}{$\begin{array}{l}\text { Quality of } \\
\text { evidence }\end{array}$} & \multicolumn{2}{|c|}{ Effect estimate (95\% CI) } & \multirow{2}{*}{ p-value } \\
\hline & & & MD & SMD & \\
\hline \multicolumn{6}{|l|}{ Ventilation } \\
\hline $\mathrm{RR}(\mathrm{bpm})$ & $58(2)$ & $\oplus \oplus \oplus \ominus^{\mathrm{a}}$ Moderate & $-2.00(-5.84,-1.84)$ & & 0.31 \\
\hline $\mathrm{V}_{\mathrm{T}}(\mathrm{L})$ & $58(2)$ & $\oplus \oplus \oplus \ominus^{\mathrm{a}}$ Moderate & $0.14(-0.09,0.38)$ & & 0.23 \\
\hline $\mathrm{V}_{\mathrm{e}}(\mathrm{L} / \mathrm{min})$ & $58(2)$ & $\oplus \oplus \oplus \ominus^{\mathrm{a}}$ Moderate & $-3.22(-10.60,4.17)$ & & 0.39 \\
\hline $\mathrm{T}_{\mathrm{i}}(\mathrm{s})$ & $58(2)$ & $\oplus \oplus \oplus \ominus^{\mathrm{a}}$ Moderate & $0.06(-0.05,0.17)$ & & 0.28 \\
\hline $\mathrm{T}_{\mathrm{e}}(\mathrm{s})$ & $58(2)$ & $\oplus \oplus \oplus \ominus^{\mathrm{a}}$ Moderate & $0.20(-0.05,0.44)$ & & 0.12 \\
\hline IC & $58(2)$ & $\oplus \oplus \oplus \ominus^{\mathrm{a}}$ Moderate & $0.09(-0.30,0.48)$ & & 0.65 \\
\hline \multicolumn{6}{|c|}{ Sensation of dyspnea } \\
\hline Dyspnea & $58(2)$ & $\oplus \oplus \oplus \ominus^{\mathrm{a}}$ Moderate & $1.02(-0.37,2.41)$ & & 0.15 \\
\hline \multicolumn{6}{|l|}{ Exercise capacity } \\
\hline HR (bpm) & $58(2)$ & $\oplus \oplus \oplus \ominus^{\mathrm{a}}$ Moderate & $-5.50(-12.65,1.64)$ & & 0.13 \\
\hline $\mathrm{VO}_{2}(\mathrm{~mL} / \mathrm{min})$ & $58(2)$ & $\oplus \oplus \oplus \ominus^{\mathrm{a}}$ Moderate & $-170.47(-312.63,-28.31)$ & & $0.02^{*}$ \\
\hline
\end{tabular}

$\mathrm{MD}$, mean difference; SMD, standard mean difference; VF, ventilatory feedback; RR, respiratory rate; $\mathrm{V}_{\mathrm{T}}$, tidal volume;

$\mathrm{V}_{\mathrm{e}}$, minute ventilation; $\mathrm{T}_{\mathrm{i}}$, inspiratory time; $\mathrm{T}_{\mathrm{e}}$, expiratory time; $\mathrm{HR}$, heart rate; $\mathrm{VO}_{2}$, oxygen consumption.

${ }^{a}$ Serious imprecision of measurements (sample size $<400$ participants).

${ }^{\mathrm{b}}$ Serious inconsistency of results $\left(\mathrm{I}^{2} \geq 50 \%\right)$.

${ }^{\mathrm{c}}$ Serious risk of bias ( $>25 \%$ of participants from study with high risk of bias).

${ }^{*} \mathrm{p}<0.05$. 
Table S4. Summary of the effects of singing compared to control group

\begin{tabular}{|c|c|c|c|c|c|}
\hline \multirow{2}{*}{ Outcome } & \multirow{2}{*}{$\begin{array}{c}\text { No. of participants } \\
\text { (studies) }\end{array}$} & \multirow{2}{*}{$\begin{array}{l}\text { Quality of } \\
\text { evidence }\end{array}$} & \multicolumn{2}{|c|}{ Effect estimate (95\% CI) } & \multirow{2}{*}{ p-value } \\
\hline & & & MD & SMD & \\
\hline \multicolumn{6}{|l|}{ Ventilation } \\
\hline Breath hold (s) & $52(2)$ & $\oplus \oplus \oplus \ominus^{\mathrm{a}}$ Moderate & $-4.89(-8.34,-1.44)$ & & $0.005^{*}$ \\
\hline Single breath & $52(2)$ & $\oplus \oplus \oplus \ominus^{\mathrm{a}}$ Moderate & $-2.93(-6.35,0.49)$ & & 0.09 \\
\hline \multicolumn{6}{|l|}{ Exercise capacity } \\
\hline ISWT (m) & $52(2)$ & $\oplus \oplus \oplus \ominus^{\mathrm{a}}$ Moderate & $-11.07(-39.38,17.24)$ & & 0.44 \\
\hline \multicolumn{6}{|l|}{ QoL } \\
\hline SF-36 PCS & $52(2)$ & $\oplus \oplus \oplus \ominus^{\mathrm{a}}$ Moderate & $12.64(5.50,19.77)$ & & $0.0005^{*}$ \\
\hline SF-36 MCS & $52(2)$ & $\oplus \oplus \oplus \ominus^{\mathrm{a}}$ Moderate & $5.42(-3.90,14.74)$ & & 0.25 \\
\hline HAD-anxiety & $52(2)$ & $\oplus \oplus \oplus \ominus^{\mathrm{a}}$ Moderate & $-1.25(-2.61,0.10)$ & & 0.07 \\
\hline HAD-sepression & $52(2)$ & $\oplus \oplus \oplus \ominus^{\mathrm{a}}$ Moderate & $-0.87(-2.16,0.42)$ & & 0.19 \\
\hline QoL & $82(3)$ & $\oplus \oplus \oplus \ominus^{\mathrm{a}}$ Moderate & & $-0.14(-0.58,0.29)$ & 0.52 \\
\hline
\end{tabular}

MD, mean difference; SMD, standard mean difference; ISWT, incremental shuttle walk test; SF-36, Short Form 36 Questionnaire; PCS, physical component summary; MCS, mental component summary; HAD, Hospital Anxiety and Depression score; QoL, quality of life.

${ }^{a}$ Serious imprecision of measurements (sample size $<400$ participants).

${ }^{\mathrm{b}}$ Serious inconsistency of results $\left(\mathrm{I}^{2} \geq 50 \%\right)$.

'Serious risk of bias ( $>25 \%$ of participants from study with high risk of bias).

${ }^{*} \mathrm{p}<0.001$.

Table S5. Summary of the effects of combined BEs compared to control group

\begin{tabular}{|c|c|c|c|c|c|}
\hline \multirow{2}{*}{ Outcome } & \multirow{2}{*}{$\begin{array}{c}\text { No. of participants } \\
\text { (studies) }\end{array}$} & \multirow{2}{*}{ Quality of evidence } & \multicolumn{2}{|c|}{ Effect estimate (95\% CI) } & \multirow{2}{*}{ p-value } \\
\hline & & & MD & SMD & \\
\hline \multicolumn{6}{|l|}{ Ventilation } \\
\hline $\mathrm{RR}$ & $111(2)$ & $\oplus \oplus \oplus \Theta^{\mathrm{a}}$ Moderate & $-5.53(-6.98,-4.09)$ & & $<0.00001^{*}$ \\
\hline $\mathrm{SpO}_{2}$ & $91(2)$ & $\oplus \oplus \oplus \ominus^{\mathrm{a}}$ Moderate & & $0.04(-0.37,0.46)$ & 0.84 \\
\hline \multicolumn{6}{|l|}{ Exercise capacity } \\
\hline $\mathrm{VO}_{2}(\mathrm{~mL} / \mathrm{min})$ & $111(2)$ & $\oplus \oplus \oplus \ominus^{\mathrm{a}}$ Moderate & $-7.31(-18.39,3.78)$ & & 0.20 \\
\hline
\end{tabular}

$\mathrm{MD}$, mean difference; $\mathrm{SMD}$, standard mean difference; $\mathrm{RR}$, respiratory rate; $\mathrm{SpO}_{2}$, oxygen saturation; $\mathrm{VO}_{2}$, oxygen consumption.

${ }^{a}$ Serious imprecision of measurements (sample size $<400$ participants).

${ }^{\mathrm{b}}$ Serious inconsistency of results $\left(\mathrm{I}^{2} \geq 50 \%\right)$.

${ }^{\mathrm{c}}$ Serious risk of bias ( $>25 \%$ of participants from study with high risk of bias).

${ }^{*} \mathrm{p}<0.001$. 
Table S6. Summary of the effects of DBE compared to control group

\begin{tabular}{|c|c|c|c|c|c|}
\hline \multirow{2}{*}{ Outcome } & \multirow{2}{*}{$\begin{array}{c}\text { No. of participants } \\
\text { (studies) }\end{array}$} & \multirow{2}{*}{$\begin{array}{l}\text { Quality of } \\
\text { evidence }\end{array}$} & \multicolumn{2}{|c|}{ Effect estimate (95\% CI) } & \multirow{2}{*}{ p-value } \\
\hline & & & MD & SMD & \\
\hline \multicolumn{6}{|l|}{ Ventilation } \\
\hline $\mathrm{RR}$ & $155(2)$ & $\oplus \oplus \ominus^{\mathrm{a}} \Theta^{\mathrm{b}}$ Low & & $-1.09(-2.19,-0.00)$ & 0.05 \\
\hline \multicolumn{6}{|c|}{ Sensation of dyspnea } \\
\hline Dyspnea & $121(2)$ & $\oplus \oplus \ominus^{\mathrm{a}} \Theta^{\mathrm{b}}$ Low & & $0.21(-0.37,0.79)$ & 0.47 \\
\hline \multicolumn{6}{|l|}{ QoL } \\
\hline SGRO score & $123(2)$ & $\oplus \oplus \Theta^{\mathrm{a}} \Theta^{\mathrm{b}}$ Low & & $0.20(-0.51,0.91)$ & 0.058 \\
\hline
\end{tabular}

MD, mean difference; SMD, standard mean difference; DBE, diaphragmatic breathing; RR, respiratory rate; SGRQ, St. George's Respiratory Questionnaire.

${ }^{a}$ Serious imprecision of measurements (sample size $<400$ participants).

${ }^{\mathrm{b}}$ Serious inconsistency of results $\left(\mathrm{I}^{2} \geq 50 \%\right)$.

${ }^{\mathrm{c}}$ Serious risk of bias ( $>25 \%$ of participants from study with high risk of bias). 